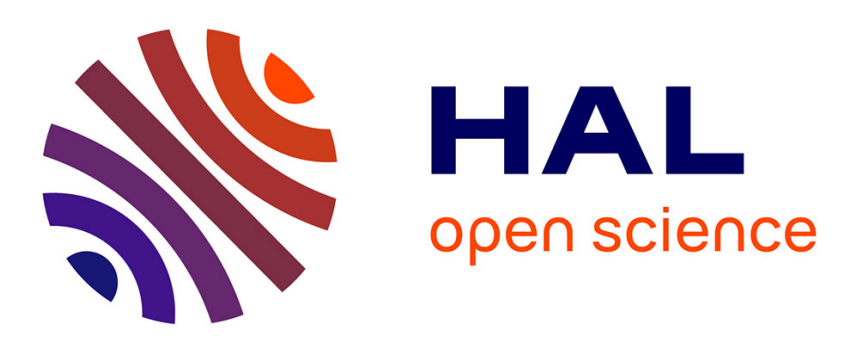

\title{
Graphene-based amplification and tuning of near-field radiative heat transfer between dissimilar polar materials
}

Riccardo Messina, Philippe Ben-Abdallah, Brahim Guizal, Mauro Antezza

\section{- To cite this version:}

Riccardo Messina, Philippe Ben-Abdallah, Brahim Guizal, Mauro Antezza. Graphene-based amplification and tuning of near-field radiative heat transfer between dissimilar polar materials. Physical Review B, 2017, 96, pp.045402. 10.1103/PhysRevB.96.045402 . hal-01557223

\section{HAL Id: hal-01557223 \\ https://hal.science/hal-01557223}

Submitted on 13 Oct 2020

HAL is a multi-disciplinary open access archive for the deposit and dissemination of scientific research documents, whether they are published or not. The documents may come from teaching and research institutions in France or abroad, or from public or private research centers.
L'archive ouverte pluridisciplinaire HAL, est destinée au dépôt et à la diffusion de documents scientifiques de niveau recherche, publiés ou non, émanant des établissements d'enseignement et de recherche français ou étrangers, des laboratoires publics ou privés. 


\title{
Graphene-based amplification and tuning of near-field radiative heat transfer between dissimilar polar materials
}

\author{
Riccardo Messina, ${ }^{1, *}$ Philippe Ben-Abdallah, ${ }^{2,3, \dagger}$ Brahim Guizal,${ }^{1, \ddagger}$ and Mauro Antezza ${ }^{1,4, \S}$ \\ ${ }^{1}$ Laboratoire Charles Coulomb, UMR 5221 CNRS-Université de Montpellier, F-34095 Montpellier, France \\ ${ }^{2}$ Laboratoire Charles Fabry, UMR 8501, Institut d'Optique, CNRS, Université Paris-Saclay, 2 Avenue Augustin Fresnel, \\ F-91127 Palaiseau Cedex, France \\ ${ }^{3}$ Department of Mechanical Engineering, Université de Sherbrooke, Sherbrooke, Quebec, Canada J1K $2 R 1$ \\ ${ }^{4}$ Institut Universitaire de France, 1 rue Descartes, F-75231 Paris Cedex 5, France
}

(Received 4 May 2017; published 5 July 2017)

\begin{abstract}
The radiative heat transfer between two dielectrics can be strongly enhanced in the near field in the presence of surface phonon-polariton resonances. Nevertheless, the spectral mismatch between the surface modes supported by two dissimilar materials is responsible for a dramatic reduction of the radiative heat flux they exchange. In the present paper we study how the presence of a graphene sheet, deposited on the material supporting the surface wave of lowest frequency, allows us to widely tune the radiative heat transfer, producing an amplification factor going up to one order of magnitude. By analyzing the Landauer energy transmission coefficients we demonstrate that this amplification results from the interplay between the delocalized plasmon supported by graphene and the surface polaritons of the two dielectrics. We finally show that the effect we highlight is robust with respect to the frequency mismatch, paving the way to an active tuning and amplification of near-field radiative heat transfer in different configurations.
\end{abstract}

DOI: 10.1103/PhysRevB.96.045402

\section{INTRODUCTION}

Improving radiative heat exchanges between two bodies separated by a gap is a longstanding problem in physics. At large separation distance (i.e., the far-field regime) energy exchanges result exclusively from propagative photons emitted by these media, and the blackbody limit [1] sets the maximum heat flux which can be exchanged between two objects. However, at subwavelength distances (i.e., the near-field regime) the situation radically changes [2,3]. Indeed, at this scale, the evanescent photons which remain confined near the surface of materials [4] are the main contributors to the heat transfer [5,6] by tunneling through the separation gap. A significant heat flux increase results from this transport [7-22]. In the presence of resonant surface modes [5], a continuum of hyperbolic modes [23], or surface Bloch waves [24], the radiative heat exchanges can drastically surpass by several orders of magnitude the prediction of Planck's blackbody theory. However, when the two media interacting are dissimilar, the spectral mismatch between their optical properties limits dramatically the amount of energy they can exchange between each other [25]. To limit this effect, composite systems made with a single or several graphene sheets have been suggested [26-49]. These systems exploit the exceptional optical properties of graphene [50,51]. More specifically, the radiative heat transfer between suspended graphene sheets has been analyzed [29,43], as well as that in configurations where graphene is deposited either on dielectric substrates [26-28,31,33,35,45,49] or on metamaterials $[36,38,39,41,42,48]$. Besides these fundamental

\footnotetext{
*riccardo.messina@umontpellier.fr

†pba@institutoptique.fr

†brahim.guizal@umontpellier.fr

§mauro.antezza@umontpellier.fr
}

developments, graphene sheets have also been considered for several applicative purposes, such as thermophotovoltaic conversion [30,32,37,40,47], thermal rectification [44], and heat transfer amplification [46].

In this paper we investigate the role that a graphene sheet can play in the near-field heat exchanges between two planar media which support two surface waves at two different frequencies in the Planck window where the near-field exchanges take place. To this aim we consider two polar materials, and we analyze, using the Landauer-like theory of radiative heat exchanges [25,52], the net heat flux exchanged between these media when a graphene sheet is deposited on one of the polar materials. We show that the presence of graphene produces, indeed, an amplification of the flux, going up to one order of magnitude in the near-field regime. This amplification can be largely tuned by acting on the graphene chemical potential, and it is related to the modification of surface modes induced by the presence of graphene. After discussing this enhancement in the case of a specific choice of two dielectrics, we also prove that this effect is robust with respect to the mismatch in resonance frequencies existing between the two substrates.

This paper is structured as follows. In Sec. II, we present our physical system, introduce the optical properties of involved materials, and recall the definition of heat flux exchanged both in the near field and in the far field between two planar media. In Sec. III we calculate this flux with respect to the separation distances between the two polar media and with respect to the chemical potential of graphene. To quantify the role played by the graphene sheet we also introduce an amplification coefficient of heat flux due to the presence of graphene, and we show that under appropriate conditions the latter can significantly amplify energy exchanges between the two polar materials despite their spectral mismatch. We also demonstrate that the heat flux can be controlled by tuning the chemical potential of graphene. Next, to get insight into 
the physical origin of the amplification we analyze in Sec. IV in the frequency-wave-vector plane the Landauer transmission coefficients, that is, the coupling efficiency of modes supported by the two media, in the presence or absence of graphene with respect to the separation distance and the doping level. We then show in Sec. V that the amplification we describe is stable with respect to the frequency mismatch between the two dielectric substrates. We finally summarize our results in Sec. VI.

\section{PHYSICAL SYSTEM}

The physical system we consider, represented in Fig. 1, is made of two parallel planar slabs of infinite thickness. We assume that slab 1 , made of zinc sulfide $(\mathrm{ZnS})$, is kept at the temperature $T_{1}=290 \mathrm{~K}$, while slab 2, made of gallium arsenide (GaAs), is kept at $T_{2}=310 \mathrm{~K}$, so that the Planck window is centered around $\lambda=10 \mu \mathrm{m}\left(\omega \simeq 1.8 \times 10^{14} \mathrm{rad} / \mathrm{s}\right)$. For the distance between the two slabs, noted with $d$, we will consider the region going from $10 \mathrm{~nm}$ to $3 \mu \mathrm{m}$ in order to explore the transition between the near and far fields. Starting from this reference configuration, we will first study the effect of one single layer of graphene deposited on the vacuum-GaAs interface, as shown in Fig. 1.

Let us now focus on the optical description of the materials involved in the problem. We describe both GaAs and $\mathrm{ZnS}$ by means of a Drude-Lorentz model,

$$
\varepsilon(\omega)=\varepsilon_{\text {inf }} \frac{\omega^{2}-\omega_{\mathrm{L}}^{2}+i \gamma \omega}{\omega^{2}-\omega_{\mathrm{T}}^{2}+i \gamma \omega},
$$

with the model parameters being [53] $\varepsilon_{\text {inf }}=5.7, \omega_{\mathrm{L}}=$ $0.66 \times 10^{14} \mathrm{rad} / \mathrm{s}, \omega_{\mathrm{T}}=0.53 \times 10^{14} \mathrm{rad} / \mathrm{s}$, and $\gamma=1.28 \times$ $10^{12} \mathrm{rad} / \mathrm{s}$ for $\mathrm{ZnS}$ and $\varepsilon_{\mathrm{inf}}=11.0, \omega_{\mathrm{L}}=0.55 \times 10^{14} \mathrm{rad} / \mathrm{s}$, $\omega_{\mathrm{T}}=0.51 \times 10^{14} \mathrm{rad} / \mathrm{s}$, and $\gamma=4.52 \times 10^{11} \mathrm{rad} / \mathrm{s}$ for GaAs. This model predicts for both materials the existence of a surface phonon-polariton resonance, having frequency $\omega_{1} \simeq$

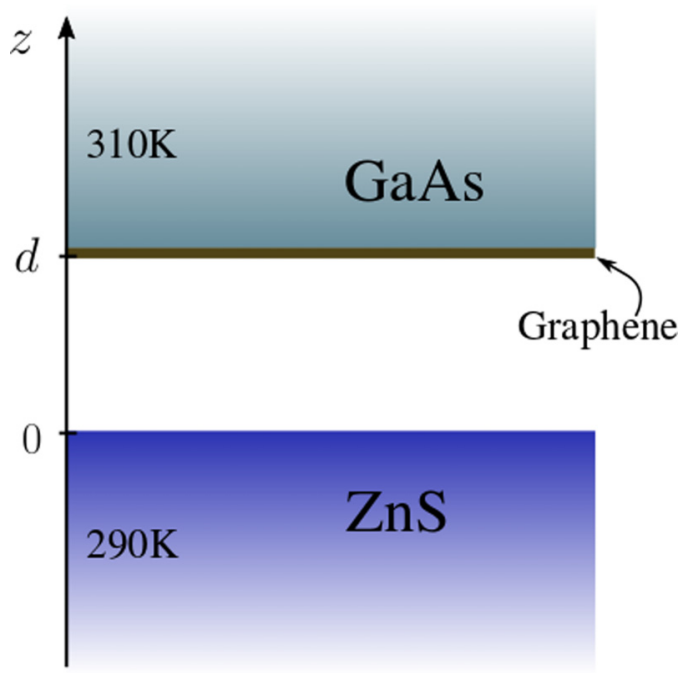

FIG. 1. Geometry of the system. Two planar slabs of infinite thickness, made of $\mathrm{ZnS}$ and GaAs, respectively, are separated by a distance $d$. A graphene sheet is deposited at $z=d$, i.e., at the vacuum-GaAs interface. The temperatures of the two slabs are fixed at $T_{1}=290 \mathrm{~K}$ and $T_{2}=310 \mathrm{~K}$ throughout the paper.
$0.65 \times 10^{14} \mathrm{rad} / \mathrm{s}$ for $\mathrm{ZnS}$ and a lower frequency, $\omega_{2} \simeq 0.55 \times$ $10^{14} \mathrm{rad} / \mathrm{s}$, for GaAs.

The optical properties of graphene will be described in terms of a two-dimensional conductivity $\sigma(\omega)$. Following Ref. [54], this can be written as a sum of intraband (Drude) and interband contributions, respectively given by

$$
\begin{aligned}
& \sigma_{D}(\omega)=\frac{i}{\omega+\frac{i}{\tau}} \frac{2 e^{2} k_{B} T}{\pi \hbar^{2}} \ln \left(2 \cosh \frac{\mu}{2 k_{B} T}\right), \\
& \sigma_{I}(\omega)=\frac{e^{2}}{4 \hbar}\left[G\left(\frac{\hbar \omega}{2}\right)+i \frac{4 \hbar \omega}{\pi} \int_{0}^{+\infty} \frac{G(\xi)-G\left(\frac{\hbar \omega}{2}\right)}{(\hbar \omega)^{2}-4 \xi^{2}} d \xi\right]
\end{aligned}
$$

where $G(x)=\sinh \left(x / k_{B} T\right) /\left[\cosh \left(\mu / k_{B} T\right)+\cosh \left(x / k_{B} T\right)\right]$. The conductivity depends explicitly on the temperature $T$ of the graphene sheet, for which we have chosen the same value, $T=310 \mathrm{~K}$, as the GaAs substrate. In addition, Eq. (3) contains the relaxation time $\tau$, which we have fixed (following Ref. [55]) to the value $\tau=10^{-13} \mathrm{~s}$. Finally, the conductivity depends on the chemical potential $\mu$, allowing us to actively tune the optical response of graphene and, in turn, the radiative heat transfer between the two structures. The model described by Eq. (2) neglects the mass-gap parameter as well as nonlocal effects [56-58].

We now need the explicit expression of the radiative heat transfer per unit area exchanged between the two structures. It is convenient to express this in the form of a Landauer decomposition [25],

$$
\varphi=\int_{0}^{\infty} \frac{d \omega}{2 \pi} \hbar \omega n_{21}(\omega) \sum_{p} \int \frac{d^{2} \mathbf{k}}{(2 \pi)^{2}} \mathcal{T}_{p}(\omega, \mathbf{k}),
$$

where $n_{\alpha \beta}(\omega)=n_{\alpha}(\omega)-n_{\beta}(\omega)$ is the difference between the two mean photon occupation numbers $n_{\alpha}(\omega)=$ $\left(\exp \left[\hbar \omega / k_{B} T_{\alpha}\right]-1\right)^{-1}$, with $\alpha=1,2$. The decomposition in Eq. (3) describes the radiative heat flux as the sum of contributions coming from each field mode, identified by the frequency $\omega$, the parallel wave vector $\mathbf{k}=\left(k_{x}, k_{y}\right)$, and the polarization $p$, which can be transverse electric (TE) or transverse magnetic (TM): each mode transports an energy $\hbar \omega$, multiplied by a transmission coefficient $\mathcal{T}_{p}(\omega, \mathbf{k})$, taking values between 0 and 1 . In the case of two parallel planar slabs, this quantity reads (the dependence on frequency and wave vector is implicit)

$$
\mathcal{T}_{p}= \begin{cases}\frac{\left(1-\left|\rho_{1, p}\right|^{2}\right)\left(1-\left|\rho_{2, p}\right|^{2}\right)}{\left|1-\rho_{1, p} \rho_{2, p} e^{2 i k_{z} d}\right|^{2}}, & k<\frac{\omega}{c}, \\ \frac{4 \operatorname{Im}\left(\rho_{1, p}\right) \operatorname{Im}\left(\rho_{2, p}\right) e^{-2 \operatorname{Im}\left(k_{z}\right) d}}{\left|1-\rho_{1, p} \rho_{2, p} e^{-2 \operatorname{Im}\left(k_{z}\right) d}\right|^{2}}, & k>\frac{\omega}{c},\end{cases}
$$

where $k_{z}=\sqrt{\omega^{2} / c^{2}-\mathbf{k}^{2}}$ is the normal component of the wave vector in vacuum, while $\rho_{i, p}$ is the reflection coefficient of body $i=1,2$ for polarization $p$. While the reflection coefficients $\rho_{1, p}$ coincide with the standard Fresnel coefficients, the quantities $\rho_{2, p}$ have to be modified in order to take into account 

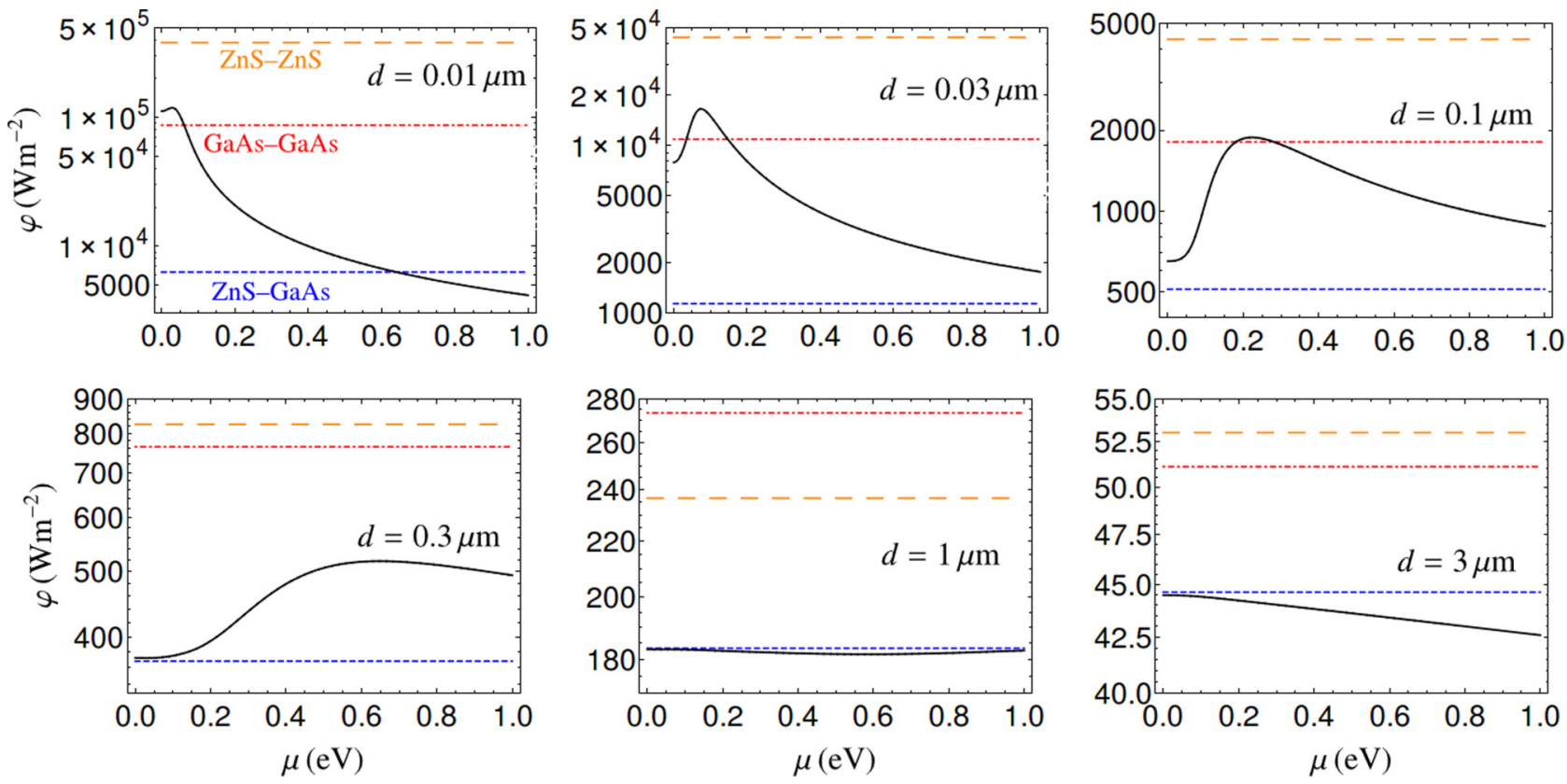

FIG. 2. Radiative heat flux $\varphi$ per unit area as a function of the graphene chemical potential $\mu$ for six different values of the distance $d$ (as indicated in each panel). The three horizontal lines in each curve correspond to three reference values in the absence of graphene: ZnS-ZnS (orange long-dashed line), GaAs-GaAs (red dot-dashed line) and ZnS-GaAs (blue short-dashed line).

the presence of a graphene sheet. They read [31]

$$
\begin{aligned}
\rho_{2, \mathrm{TE}} & =\frac{k_{z}-k_{z}^{(2)}-\mu_{0} \sigma(\omega) \omega}{k_{z}+k_{z}^{(2)}+\mu_{0} \sigma(\omega) \omega}, \\
\rho_{2, \mathrm{TM}} & =\frac{\varepsilon_{2}(\omega) k_{z}-k_{z}^{(2)}+\frac{\sigma(\omega) k_{z} k_{z}^{(2)}}{\varepsilon_{0} \omega}}{\varepsilon_{2}(\omega) k_{z}+k_{z}^{(2)}+\frac{\sigma(\omega) k_{z} k_{z}^{(2)}}{\varepsilon_{0} \omega}},
\end{aligned}
$$

where $k_{z}^{(2)}=\sqrt{\varepsilon_{2}(\omega) \omega^{2} / c^{2}-\mathbf{k}^{2}}$ is the normal component of the wave vector in medium 2 . Since $\rho_{2, p}$ contains the graphene conductivity, it depends on the temperature $T_{2}$ and on the chemical potential $\mu$.

\section{AMPLIFICATION AND TUNING OF RADIATIVE HEAT TRANSFER}

We now have all the ingredients needed to analyze the heat transfer given by Eq. (3) as a function of both the distance $d$ and the graphene chemical potential $\mu$. As far as the former is concerned, we are going to explore the region $d \in[10 \mathrm{~nm}, 3 \mu \mathrm{m}]$, fully catching the near-field behavior and the transition toward distances at which the far-field is relevant. Concerning the chemical potential, we are going to restrict our analysis to the range $\mu \in[0,1] \mathrm{eV}$, containing physically accessible values.

We show our first set of results in Fig. 2, where the flux $\varphi$ is plotted as a function of the chemical potential $\mu$ for six different values of the distance $d$. In each panel, the $\mu$ depending flux is compared with three reference values in the absence of graphene, i.e., the configurations GaAs-GaAs and $\mathrm{ZnS}-\mathrm{ZnS}$ of equal dielectrics as well as the scenario $\mathrm{ZnS}-\mathrm{GaAs}$, obtained by just removing the graphene sheet.

A first glimpse of the six curves already gives an idea of the possibilities offered by the presence of graphene in terms of manipulation of the radiative flux. In particular, we observe not only that the flux can be monotonic or not with respect to $\mu$ depending on the distance considered but also that the chemical potentials maximizing or minimizing the transfer are also functions of $d$. As a general feature, we note, however, that (at least in the window of $\mu$ taken into account) approaching the far field reduces the degree of variation of the flux with respect to $\mu$.

It is also instructive to consider the three reference values. We first remark that, not surprisingly, the values corresponding to couples of equal dielectrics always give a flux much higher than the configuration $\mathrm{ZnS}-\mathrm{GaAs}$, characterized by a surfaceresonance frequency mismatch. More interestingly, for some values of the distance, tuning the chemical potential allows us to go beyond the value of the flux corresponding to two GaAs substrates. This proves that the presence of graphene is not only able to permit a large variation and amplification of the flux through its chemical potential but also to fully compensate the mismatch between the resonance frequencies of the two dielectrics.

This feature is more manifest in the complementary plot given in Fig. 3, where the flux is shown as a function of the distance $d$ for five different values of the chemical potential. We first confirm that the largest possible tuning (and amplification) is realized at the smallest distance, while all the curves corresponding to different values of $\mu$ converge on each other and on the configuration corresponding to the absence of graphene (blue dashed curve) when moving to the far field. Moreover, in agreement with what was observed before, the different solid lines cross each other, showing that for each $d$ the highest and lowest fluxes are realized for different chemical potentials. Finally, we clearly highlight two regions of distances where even the flux between two GaAs substrates is surpassed. 


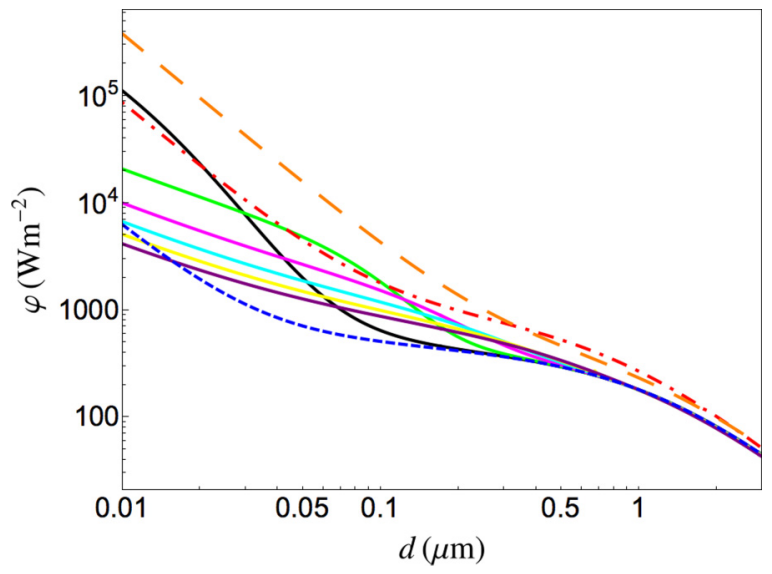

FIG. 3. Radiative flux $\varphi$ per unit area as a function of the distance $d$ for six different values of the graphene chemical potential $\mu$. From top to bottom at $d=10 \mathrm{~nm}$, the curves correspond to $\mu=0 \mathrm{eV}$ (black), $0.2 \mathrm{eV}$ (green), $0.4 \mathrm{eV}$ (magenta), $0.6 \mathrm{eV}$ (cyan), $0.8 \mathrm{eV}$ (yellow), and $1.0 \mathrm{eV}$ (purple). We also show three lines corresponding to reference values in the absence of graphene: $\mathrm{ZnS}-\mathrm{ZnS}$ (orange long-dashed line), GaAs-GaAs (red dot-dashed line) and $\mathrm{ZnS}$-GaAs (blue short-dashed line).

Following the results presented so far, it is interesting to give an overall image of the possibilities offered by the presence of graphene in terms of tuning and amplification of the flux. In this respect, two complementary views are possible. On the one hand, the ratio between the maximum and minimum values of $\varphi$ (with respect to the chemical potential $\mu$ ) can be plotted versus the distance $d$ : this quantity tells us how much we can tune the flux by externally acting on the chemical potential. This ratio corresponds to the red dashed line in Fig. 4. On the other hand, one can calculate the ratio between

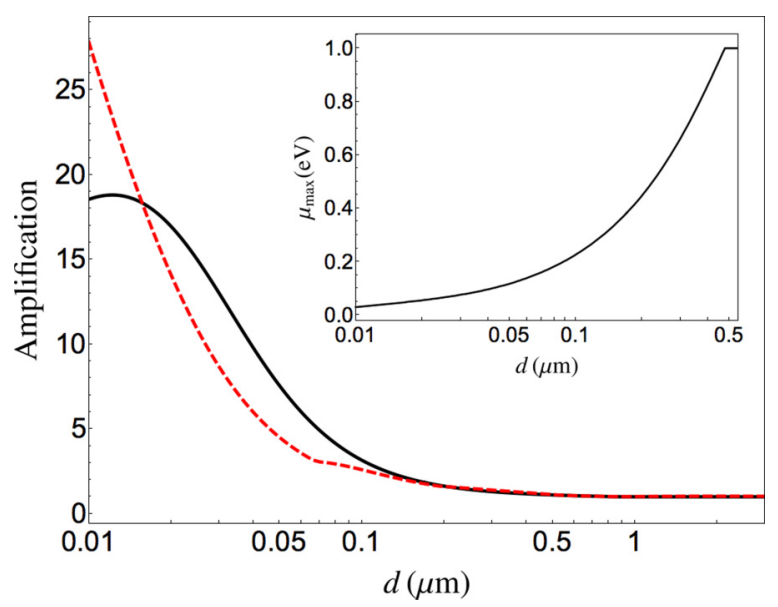

FIG. 4. Radiative-heat-flux amplification factor with respect to the graphene chemical potential $\mu$ as a function of the distance $d$. The red dashed curve corresponds to the ratio between the highest and lowest values of the flux in the presence of graphene, while the black curve corresponds to the ratio between the highest value of $\varphi$ in the presence of graphene and the flux in the $\mathrm{ZnS}-\mathrm{GaAs}$ configuration without graphene. In the inset the chemical potential $\mu_{\max }$ realizing the maximum flux in the presence of graphene is shown as a function of the distance $d$. the maximum value of the flux with respect to $\mu$ and the reference value in the $\mathrm{ZnS}-\mathrm{GaAs}$ configuration, i.e., in the absence of graphene. This second ratio describes how the presence of graphene is able to amplify the standard radiative flux between two dissimilar dielectrics by compensating the mismatch between the two different resonance frequencies. This quantity corresponds to the black line in Fig. 4. The analysis of these two curves shows that in both cases we have a remarkable amplification factor which can go beyond one order of magnitude. More specifically, Fig. 4 clearly shows that the effect we highlight is a near-field effect. As a matter of fact, starting from $d=500 \mathrm{~nm}$, i.e., when moving toward the far-field region, the two curves join each other and tend to 1 , which means an almost flat value of $\varphi$ as a function of $\mu$, in agreement with the last panels of Fig. 2. The inset in Fig. 4 shows the value $\mu_{\max }$ of the chemical potential realizing the maximum value of the flux. This quantity is plotted for $d \lesssim$ $500 \mathrm{~nm}$, i.e., in the region of distances showing a significant amplification. The curve shows that the value $\mu_{\max }$ saturates for $d \simeq 500 \mathrm{~nm}$ at the maximum value of $1 \mathrm{eV}$ imposed in our calculation. One must therefore bear in mind that the two amplification curves shown in the main part of Fig. 4 are also influenced by this choice. We will comment further on this point in the discussion of the transmission coefficients given below.

\section{TRANSMISSION COEFFICIENT AND SPECTRAL FLUX}

To get more insight into the physics behind this tuning and amplification of radiative heat transfer we now focus on the analysis of the Landauer transmission coefficient $\mathcal{T}_{p}(\omega, \mathbf{k})$ for several configurations both in the absence and in the presence of graphene. As stated above, this quantity, always between 0 and 1 , describes the rate of participation of the mode having polarization $p$, frequency $\omega$, and wave vector $\mathbf{k}$ to the energy exchange. In the following we focus only on TM polarization since it is well known that it is the one mainly contributing to the amplification of radiative heat transfer in the near field [5]. To start with, we focus on the distance $d=20 \mathrm{~nm}$, well within the near-field region, and we show in Fig. 5 the Landauer coefficients associated with the three standard dielectricdielectric configurations, namely, GaAs-GaAs, $\mathrm{ZnS}-\mathrm{ZnS}$, and $\mathrm{ZnS}-\mathrm{GaAs}$. Figures 5(a) and 5(b) show a scenario typical in the literature for near-field radiative heat transfer. We see two branches (symmetric and antisymmetric) of surface modes, converging to a horizontal asymptote corresponding to the frequencies of the surface resonances of the two materials. We observe that the branches associated with GaAs are thinner and are limited to smaller values of $k$. This stems from the fact that GaAs has smaller losses than $\mathrm{ZnS}$, which is manifest from the parameters given after Eq. (1). Figure 5(c) shows the transmission coefficient for the $\mathrm{ZnS}-\mathrm{GaAs}$ configuration. We immediately see that, although the resonance frequencies are relatively close to each other, the mismatch produces a remarkable decoupling, reducing dramatically the number of modes effectively participating in the exchange and thus the total integrated flux.

We now turn our attention to the transmission coefficients in the presence of graphene by considering in Fig. 6 four 

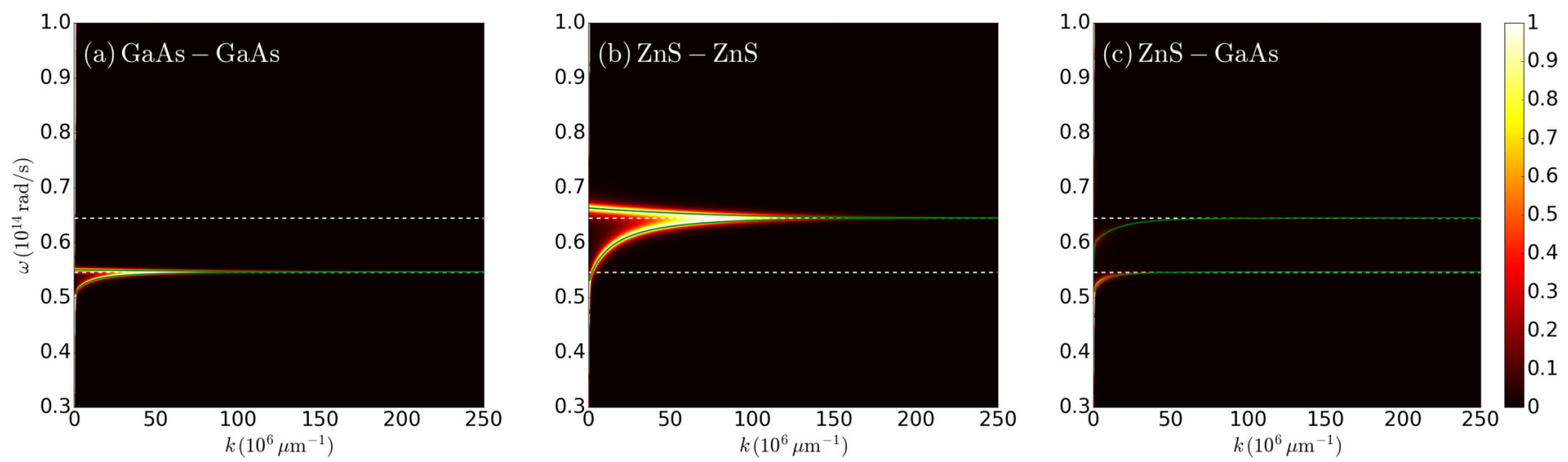

FIG. 5. Landauer transmission coefficient $\mathcal{T}_{p}(\omega, \mathbf{k})$ in the $(k, \omega)$ plane for the three reference dielectric-dielectric configurations in the absence of graphene for $d=20 \mathrm{~nm}$ in TM polarization: (a) GaAs-GaAs, (b) ZnS-ZnS, and (c) ZnS-GaAs. The horizontal lines correspond to the resonance frequencies of $\mathrm{GaAs}$ and $\mathrm{ZnS}$. The green lines describe the dispersion relation of the cavity surface modes.

different values of the chemical potential, namely, $\mu=0 \mathrm{eV}$, $0.05 \mathrm{eV}$ (close to the value $\mu_{\max }$ realizing the highest flux for $d=20 \mathrm{~nm}), 0.5 \mathrm{eV}$, and $1 \mathrm{eV}$. As a general remark, by comparing these plots with Fig. 5(c), we observe that all the considered values of $\mu$ clearly increase the number of modes contributing to the flux. In addition, we see that for $\mu=0.05 \mathrm{eV} \simeq \mu_{\max }$ the presence of graphene creates a region of modes with $\omega \simeq \omega_{1}$ (the resonance frequency of $\mathrm{ZnS}$ ) and relatively high wave vector with a non-negligible value of $\mathcal{T}_{p}(\omega, \mathbf{k})$. In fact, it is important to observe not only the increased number of modes but also their typical wave vector since each mode participates in the heat transfer between two planar slabs through an additional factor $k$, namely, from the Jacobian when moving to polar coordinates in the $\left(k_{x}, k_{y}\right)$
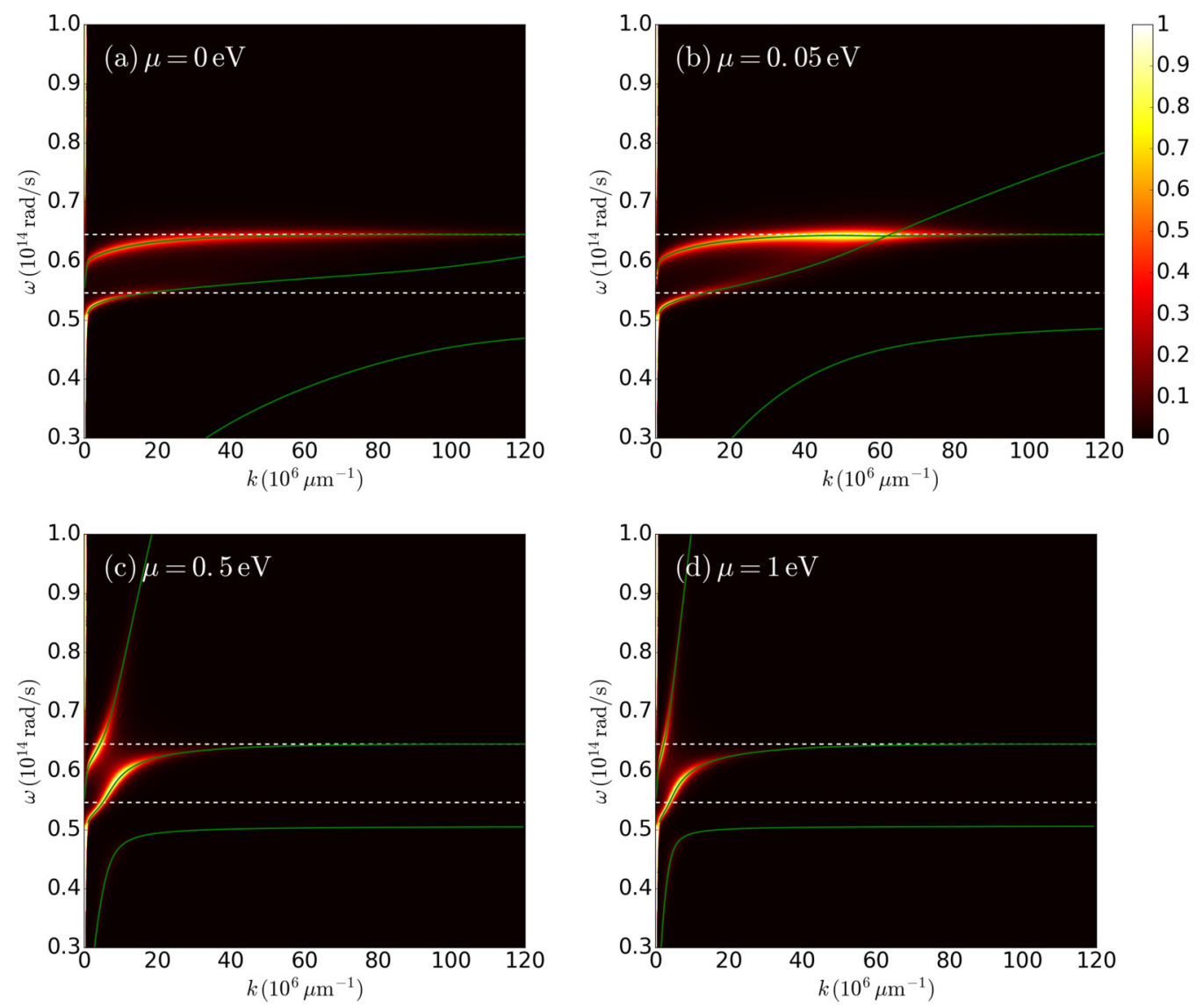

FIG. 6. Landauer transmission coefficient $\mathcal{T}_{p}(\omega, \mathbf{k})$ in the $(k, \omega)$ plane in the presence of graphene for $d=20 \mathrm{~nm}$ in TM polarization. The four panels correspond to different values of the chemical potential: (a) $\mu=0 \mathrm{eV}$, (b) $\mu \simeq \mu_{\max }=0.05 \mathrm{eV}$, (c) $\mu=0.5 \mathrm{eV}$, and (d) $\mu=1 \mathrm{eV}$. The horizontal lines correspond to the resonance frequencies of GaAs and $\mathrm{ZnS}$. The green lines describe the dispersion relation of the cavity surface modes. 

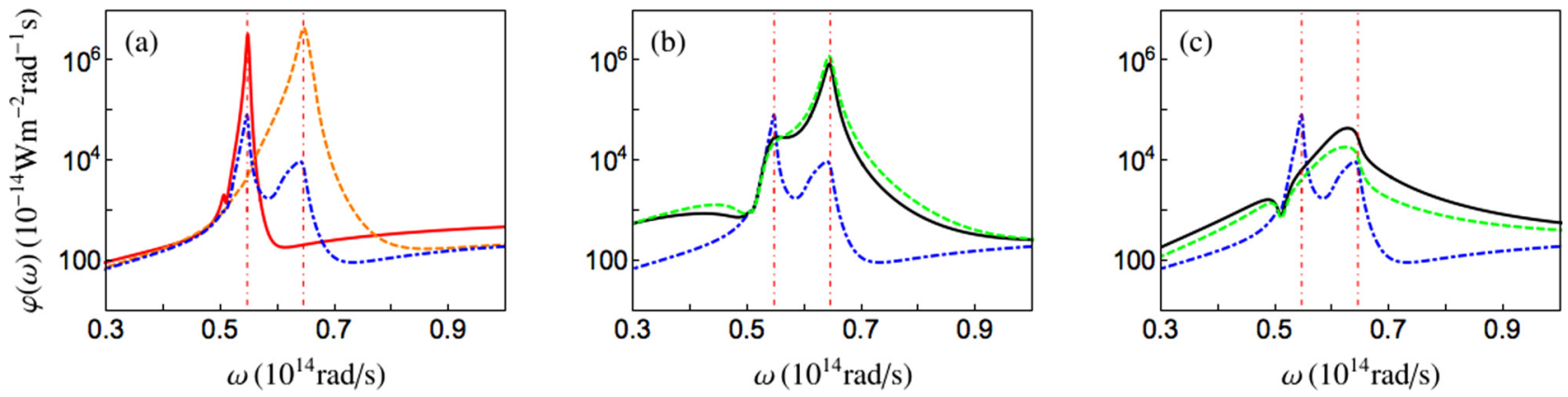

FIG. 7. Spectral flux $\varphi(\omega)$ for $d=20 \mathrm{~nm}$ for several different configurations. (a) shows three standard dielectric-dielectric configurations: GaAs-GaAs (red curve), ZnS-ZnS (orange dashed curve), and ZnS-GaAs (blue dot-dashed curve). This last curve is compared in (b) and (c) to spectral fluxes in the presence of graphene. In (c) we have $\mu=0$ (black solid line) and $\mu=0.05 \mathrm{eV}$ (green dashed line), while in (b) $\mu=0.5 \mathrm{eV}$ (black solid line) and $\mu=1 \mathrm{eV}$ (green dashed line) are shown. In the three panels the vertical red dot-dashed lines correspond to the resonances $\omega_{1}$ and $\omega_{2}$ of $\mathrm{ZnS}$ and $\mathrm{GaAs}$, respectively.

plane. For higher values of $\mu$ [Figs. 6(b) and 6(c)] the branches of resonant modes manifestly move toward smaller values of $k$, reducing the total effect.

This transition as a function of $\mu$ and in particular the existence of an optimum chemical potential $\mu_{\max }$ can be explained on the basis of the optical properties of graphene. It is well known that a suspended sheet of graphene has a delocalized surface resonance mode in TM polarization whose dispersion relation does not have a horizontal asymptote (as in the case of phonon-polaritons for dielectrics and plasmons for metals) but behaves as $\sqrt{k}$ for small wave vectors. As discussed, for instance, in Ref. [31], when a graphene sheet is deposited on a dielectric substrate supporting a phononpolariton resonance, we have a strong coupling between the two surface resonances, producing two noncrossing branches. The one at higher frequencies inherits the $\sqrt{k}$ behavior typical of graphene. We see a trace of this in the transmission coefficient shown in Fig. 6(b) corresponding to optimum heat transfer. Starting with a simplified analysis, we can say that the lower branch, associated with GaAs alone, which would have without graphene a horizontal asymptote at the GaAs resonance frequency, is shifted thanks to the presence of graphene toward higher frequencies, and thanks to the positive $d \omega / d k$ derivative inherited from the graphene surface mode, it is now able to cross the branch associated with $\mathrm{ZnS}$, producing the region of highly efficient modes evident in the plot. According to this first analysis, we would be tempted to state that a lower value of the chemical potential, leading to a lower $d \omega / d k$ derivative of the dispersion relation, would produce an even larger flux since it would produce the discussed coupling at even larger values of $k$, contributing more flux. Nevertheless, this statement ignores the fact that the modes effectively participating in the radiative heat transfer strongly depend on the distance $d$ through the exponential factor $e^{-2 \operatorname{Im}\left(k_{z}\right) d}$ in the evanescent region [see second line of Eq. (4)]. Thus, the optimal $\mu$ is, within this simplified approach, the one producing the coupling between the two branches at the highest $k$ participating in the energy exchange, roughly scaling as $1 / d$. This explains why the optimal chemical potential increases with the distance $d$ (thus reducing the $k$ at which the coupling is produced), as shown in the inset of Fig. 4. This analysis also explains why this effect basically exists only in the near field since only in this regime can high values of the wave vector be explored and exploited.

This view is further confirmed by the analysis of the dispersion relations of the cavity modes, given by the green lines in Figs. 5 and 6. These are obtained as the poles of the determinant of the scattering matrix of the cavity, coinciding with the zeros of the denominator of the transmission coefficient given in Eq. (4). In the four panels of Fig. 6, we clearly see the two lower branches coming from the strong coupling between the individual modes of GaAs and graphene. We observe that, as $\mu$ increases, so does the derivative $d \omega / d k$ of the one at higher frequency. This branch crosses the one describing the surface mode of $\mathrm{ZnS}$ at the optimal wave vector for $\mu=0.05 \mathrm{eV}$. For higher values of the chemical potential [see Fig. 6(c) and 6(d)], we observe the appearance of a further strong coupling between the graphene-GaAs mode and that of $\mathrm{ZnS}$, with an increased mode participation taking place at smaller $k$, thus producing a smaller radiative flux, as discussed above.

It is interesting to see the effect of varying the chemical potential on the spectral flux $\varphi(\omega)$, defined by the relation

$$
\varphi=\int_{0}^{+\infty} d \omega \varphi(\omega) .
$$

Figure 7(a) shows the spectral fluxes corresponding to the three dielectric-dielectric configurations. The quasimonochromatic flux typical of near-field transfer between equal materials is manifest for GaAs-GaAs and $\mathrm{ZnS}-\mathrm{ZnS}$, while the mixed configuration, ZnS-GaAs, shows a much broader and lower spectral flux. Figure 7(b) shows that the lowest values of $\mu$ allow us to tailor the spectral flux by creating a peak around the $\mathrm{ZnS}$ resonance frequency which considerably approaches that of the $\mathrm{ZnS}-\mathrm{ZnS}$ scenario. Also, the spectral flux is broader in this case as a result of the $\sqrt{k}$ behavior coming from the presence of graphene. Finally, the highest values of $\mu$ give, as shown in Fig. 7(c), an even broader spectral flux at the expense of a reduced peak flux at the resonance frequency.

\section{ROBUSTNESS WITH RESPECT TO FREQUENCY MISMATCH}

Our analysis has revealed so far that graphene is able not only to modulate the radiative heat flux between the 


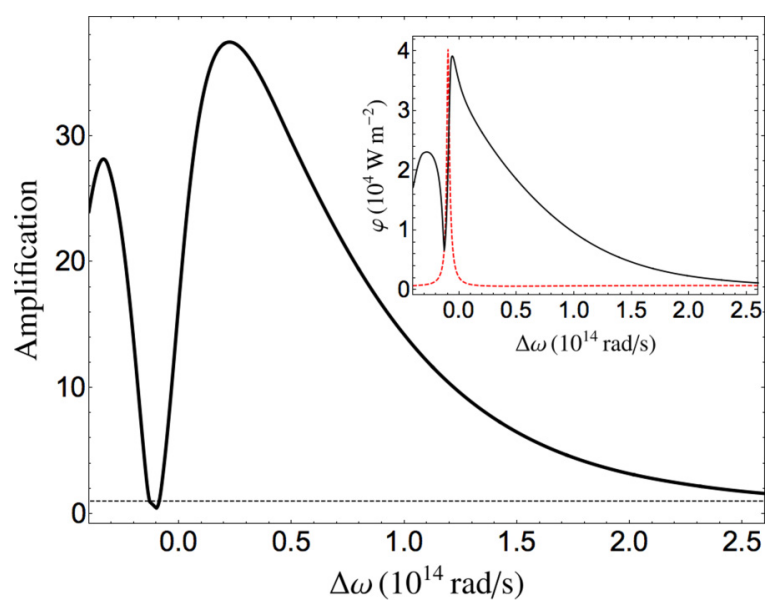

FIG. 8. Ratio between the highest flux in the presence of graphene and the one in the absence of graphene as a function of a frequency shift $\Delta \omega$ imposed on parameters $\omega_{\mathrm{L}}$ and $\omega_{\mathrm{T}}$ of $\mathrm{ZnS}$. The horizontal dashed line corresponds to amplification equal to 1 . In the inset, the flux in the absence of graphene (red dashed line) and the one in the presence of graphene (black solid line) are shown as a function of $\Delta \omega$.

two semi-infinite substrates but also to fully compensate for the frequency mismatch between the two surface resonances. Nevertheless, this study has been performed for a specific choice of the two dielectrics, corresponding to a frequency mismatch $\omega_{1}-\omega_{2} \simeq 0.1 \times 10^{14} \mathrm{rad} / \mathrm{s}$. The aim of this section is to study how the tuning and amplification highlighted so far are robust with respect to the frequency mismatch between the two dielectrics. We expect the presence of graphene to have a negative effect in the case of identical dielectrics (and thus of perfect match of surface-resonance frequency), but how the possible amplification depends on the mismatch is not clear. To this aim, we perform a parametric study in which we artificially modify the Drude-Lorentz model given in Eq. (1) describing the optical properties of $\mathrm{ZnS}$ by adding a frequency shift $\Delta \omega$ to both $\omega_{\mathrm{L}}$ and $\omega_{\mathrm{T}}$. While representing a theoretical study (since a given $\Delta \omega$ does not necessarily represent a real material and the dissipation rate is kept constant), this analysis still gives an indication of the existence and extent of the effect considered here as a function of the frequency mismatch. Based on our definition of $\Delta \omega$, the material corresponding to $\Delta \omega=-0.1 \times 10^{14} \mathrm{rad} / \mathrm{s}$ implies a surface resonance matching that of GaAs.

In our analysis we fix the distance to the value $d=20 \mathrm{~nm}$ and study the amplification (defined as the ratio between the and best possible flux in the presence of graphene and that in the absence of graphene, the one corresponding to the black solid line in Fig. 4) as a function of the frequency shift $\Delta \omega$. The results are shown in Fig. 8. We first observe that for $\Delta \omega=0$ we find an amplification factor close to 17 , already shown in Fig. 4 for $d=20 \mathrm{~nm}$. The transition from $\Delta \omega=0$ to $\Delta \omega=$ $-0.1 \times 10^{14} \mathrm{rad} / \mathrm{s}$ shows, as expected, a dramatic reduction of the amplification factor. More specifically, when the frequency shift produces a match between the two individual resonance frequencies, the amplification goes below 1, showing that the presence of graphene is only able to reduce the heat flux in this case. More interestingly, Fig. 8 shows that, apart from a narrow range of $\Delta \omega$, the amplification shows a high value even for relatively high values of frequency mismatch, proving that this tuning and amplification effect is robust with respect to the choice of materials. This robustness can be understood in more detail by analyzing the inset of Fig. 8, where the flux in the absence of graphene (red dashed line) the optimized one in the presence of graphene (black solid line), i.e., the ones whose ratio gives the amplification factor, are shown. The flux in the absence of graphene shows a very narrow peak as a function of $\Delta \omega$ : this confirms that the wellknown near-field amplification in the presence of two surface resonances is extremely sensitive to their matching. On the contrary, for a given mismatch $\Delta \omega$, there exists a chemical potential optimizing the flux: as a result, the optimized flux in the presence of graphene remains comparable to the one corresponding to $\Delta \omega=0$ for a large range of frequency shifts. Because of the limitation imposed on the values of the chemical potential, the amplification tends to 1 for high values of $\Delta \omega$.

\section{CONCLUSIONS}

We have demonstrated that a graphene sheet can be used as a relay between two dissimilar polar materials interacting in the near field in order to tune and to magnify the radiative heat flux they exchange through the surface phonon-polariton tunneling. This effect results from a coupling of the surface plasmon of graphene with the surface polaritons characterizing the two dielectrics. A direct consequence of this coupling is an increase in the number of modes which contribute to the net flux exchanged between the two materials. More specifically, we have shown that in the near field an optimized choice of the chemical potential is able to produce a flux amplification going beyond one order of magnitude. After discussing this effect in the specific case of GaAs and $\mathrm{ZnS}$, we show that this amplification is robust with respect to the frequency mismatch between surface resonances. Our results broaden further the interest of using graphene in dielectric-dielectric scenarios in order to actively tune radiative heat transfer.
[1] M. Planck, The Theory of Heat Radiation (Dover, New York, 2011).

[2] S. Rytov, Y. Kravtsov, and V. Tatarskii, Principles of Statistical Radiophysics (Springer, Berlin, 1989), Vol. 3.

[3] D. Polder and M. van Hove, Phys. Rev. B 4, 3303 (1971).

[4] W. Eckhardt, Z. Phys. B 46, 85 (1982).
[5] K. Joulain, J.-P. Mulet, F. Marquier, R. Carminati, and J.-J. Greffet, Surf. Sci. Rep. 57, 59 (2005).

[6] A. I. Volokitin and B. N. J. Persson, Rev. Mod. Phys. 79, 1291 (2007).

[7] C. Hargreaves, Phys. Lett. A 30, 491 (1969).

[8] A. Kittel, W. Müller-Hirsch, J. Parisi, S.-A. Biehs, D. Reddig, and M. Holthaus, Phys. Rev. Lett. 95, 224301 (2005). 
[9] A. Narayanaswamy, S. Shen, and G. Chen, Phys. Rev. B 78, 115303 (2008).

[10] L. Hu, A. Narayanaswamy, X. Chen, and G. Chen, Appl. Phys. Lett. 92, 133106 (2008).

[11] S. Shen, A. Narayanaswamy, and G. Chen, Nano Lett. 9, 2909 (2009).

[12] E. Rousseau, A. Siria, G. Joudran, S. Volz, F. Comin, J. Chevrier, and J.-J. Greffet, Nat. Photonics 3, 514 (2009).

[13] R. S. Ottens, V. Quetschke, S. Wise, A. A. Alemi, R. Lundock, G. Mueller, D. H. Reitze, D. B. Tanner, and B. F. Whiting, Phys. Rev. Lett. 107, 014301 (2011).

[14] T. Kralik, P. Hanzelka, V. Musilova, A. Srnka, and M. Zobac, Rev. Sci. Instrum. 82, 055106 (2011).

[15] T. Kralik, P. Hanzelka, M. Zobac, V. Musilova, T. Fort, and M. Horak, Phys. Rev. Lett. 109, 224302 (2012).

[16] P. J. van Zwol, L. Ranno, and J. Chevrier, Phys. Rev. Lett. 108, 234301 (2012).

[17] P. J. van Zwol, S. Thiele, C. Berger, W. A. de Heer, and J. Chevrier, Phys. Rev. Lett. 109, 264301 (2012).

[18] B. Song, Y. Ganjeh, S. Sadat, D. Thompson, A. Fiorino, V. Fernández-Hurtado, J. Feist, F. J. Garcia-Vidal, J. C. Cuevas, P. Reddy, and E. Meyhofer, Nat. Nanotechnol. 10, 253 (2015).

[19] K. Kim, B. Song, V. Fernández-Hurtado, W. Lee, W. Jeong, L. Cui, D. Thompson, J. Feist, M. T. Homer Reid, F. J. GarciaVidal, J. C. Cuevas, E. Meyhofer, and P. Reddy, Nature (London) 528, 387 (2015).

[20] R. St-Gelais, L. Zhu, S. Fan, and M. Lipson, Nat. Nanotechnol. 11, 515 (2016).

[21] K. Kloppstech, N. Könne, S.-A. Biehs, A. W. Rodriguez, L. Worbes, D. Hellmann, and A. Kittel, Nat. Commun. 8, 14475 (2017).

[22] J. I. Watjen, B. Zhao, and Z. M. Zhang, Appl. Phys. Lett. 109, 203112 (2016).

[23] S.-A. Biehs, M. Tschikin, and P. Ben-Abdallah, Phys. Rev. Lett. 109, 104301 (2012).

[24] P. Ben-Abdallah, K. Joulain, and A. Pryamikov, Appl. Phys. Lett. 96, 143117 (2010).

[25] P. Ben-Abdallah and K. Joulain, Phys. Rev. B 82, 121419(R) (2010).

[26] B. N. J. Persson and H. Ueba, J. Phys. Condens. Matter 22, 462201 (2010).

[27] A. I. Volokitin and B. N. J. Persson, Phys. Rev. B 83, 241407(R) (2011).

[28] V. B. Svetovoy, P. J. van Zwol, and J. Chevrier, Phys. Rev. B 85, 155418 (2012).

[29] O. Ilic, M. Jablan, J. D. Joannopoulos, I. Celanovic, H. Buljan, and M. Soljačić, Phys. Rev. B 85, 155422 (2012).
[30] O. Ilic, M. Jablan, J. D. Joannopoulos, I. Celanovic, H. Buljan, and M. Soljačić, Opt. Express 20, A366 (2012).

[31] R. Messina, J. P. Hugonin, J.-J. Greffet, F. Marquier, Y. De Wilde, A. Belarouci, L. Frechette, Y. Cordier, and P. Ben-Abdallah, Phys. Rev. B 87, 085421 (2013).

[32] R. Messina and P. Ben-Abdallah, Sci. Rep. 3, 1383 (2013).

[33] M. Lim, S. S. Lee, and B. J. Lee, Opt. Express 21, 22173 (2013).

[34] A. D. Phan, S. Shen, and L. M. Woods, J. Phys. Chem. Lett. 4, 4196 (2013).

[35] X. L. Liu and Z. Zhang, Appl. Phys. Lett. 104, 251911 (2014).

[36] X. Liu, R. Z.Zhang, and Z. Zhang, ACS Photonics 1, 785 (2014).

[37] V. B. Svetovoy and G. Palasantzas, Phys. Rev. Appl. 2, 034006 (2014).

[38] D. Drosdoff, A. D. Phan, and L. M. Woods, Adv. Opt. Mater. 2, 1038 (2014).

[39] R. Z. Zhang, X. Liu, and Z. M. Zhang, AIP Adv. 5, 053501 (2015).

[40] M. Lim, S. Jin, S. S. Lee, and B. J. Lee, Opt. Express 23, A240 (2015).

[41] J.-Y. Chang, Y. Yang, and L. Wang, J. Quant. Spectrosc. Radiat. Transfer 184, 58 (2016).

[42] J. Song and Q. Cheng, Phys. Rev. B 94, 125419 (2016).

[43] G. Yin, J. Yang, and Y. Ma, Appl. Phys. Express 9, 122001 (2016).

[44] Z. Zheng, X. Liu, A. Wang, and Y. Xuan, Int. J. Heat Mass Transfer 109, 63 (2017).

[45] B. Zhao and Z. M. Zhang, ASME J. Heat Transfer 139, 022701 (2017).

[46] H. Simchi, J. Appl. Phys. 121, 094301 (2017).

[47] M. Lim, S. S. Lee, and B. J. Lee, J. Quant. Spectrosc. Radiat. Transfer 197, 84 (2017).

[48] Q. Zhao, T. Zhou, T. Wang, W. Liu, J. Liu, T. Yu, Q. Liao, and N. Liu, J. Phys. D 50, 145101 (2017).

[49] K. Shi, F. Bao, and S. He, ACS Photonics 4, 971 (2017).

[50] A. K. Geim and K. S. Novoselov, Nat. Mater. 6, 183 (2007).

[51] A. K. Geim, Science 324, 1530 (2009).

[52] S.-A. Biehs, E. Rousseau, and J.-J. Greffet, Phys. Rev. Lett. 105, 234301 (2010).

[53] Handbook of Optical Constants of Solids, edited by E. Palik (Academic, New York, 1998).

[54] L. A. Falkovsky, J. Phys. Conf. Ser. 129, 012004 (2008).

[55] M. Jablan, H. Buljan, and M. Soljačić, Phys. Rev. B 80, 245435 (2009).

[56] W. Wang and J. M. Kinaret, Phys. Rev. B 87, 195424 (2013).

[57] T. Stauber, J. Phys. Condens. Matter 26, 123201 (2014).

[58] G. L. Klimchitskaya and V. M. Mostepanenko, Phys. Rev. B 94, 195405 (2016). 